Insights Imaging (2012) 3 (Suppl 1):S367-S374

DOI 10.1007/s13244-012-0160-5

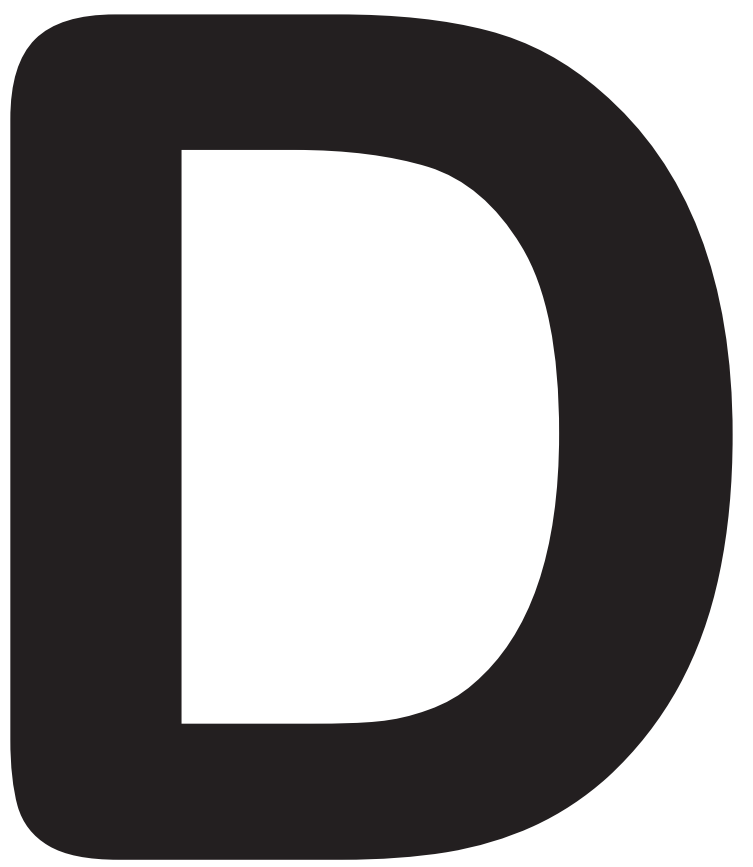

\title{
Satellite Symposia
}




\section{Thursday, March 1}

$10: 30-11: 30$

Studio 2012

\section{organised by Siemens Healthcare}

\section{SY 1}

\section{D tomosynthesis: opportunities for breast cancer imaging}

Moderator:

T. Mertelmeier; Erlangen/DE

Introduction

T. Mertelmeier; Erlangen/DE

3D breast tomosynthesis: a problem solver for difficult cases in clinical routine

J. Barkhausen; Lübeck/DE

Breast tomosynthesis: a feasible breast cancer screening modality? S. Zackrisson; Malmö/SE

3D breast tomosynthesis and contrast enhanced dual energy: a prototype report

T.H. Helbich; Vienna/AT

Panel discussion

\section{organised by SuperSonic Imagine}

\section{SY 2}

Programme not available by date of print

\section{organised by Siemens Healthcare}

\section{SY 3}

\section{Imaging for breast cancer therapy planning, execution and control}

Moderator:

J. Barkhausen; Lübeck/DE

\section{Introduction}

J. Barkhausen; Lübeck/DE

\section{D breast ultrasound: a poor man's MRI?}

M.J.C.M. Rutten; 's-Hertogenbosch/NL

Automated 3D breast ultrasound is a new technique, which scans the breast automatically and almost entirely. Data sets can be stored and are available for 3D cross correlating review. This new technique eliminates significantly the subjectivity of conventional ultrasound, disconnects US data acquisition and assessment, facilitates the possibility of double reading, presurgical workup planning, review of follow-up studies and the application of computer aided detection. Automated 3D ultrasound can be implemented in the clinical radiological setting and possibly in a personalized screening program for breast cancer.

Learning Objectives:

1. To become familiar with the $3 \mathrm{D}$ breast ultrasound technique and scanning protocols.

2. To comprehend the indications and limitations of 3D breast ultrasound.

3. To become familiar with the diagnostic accuracy of 3D breast ultrasound and to appreciate the potential added value for breast cancer screening.

4. To highlight novel developments in 3D breast US, such as computer aided detection.
Potential role of digital breast tomosynthesis (DBT) in breast cancer diagnosis

N. Uchiyama; Tokyo/JP

In this lecture, the author addresses the potential role of DBT in comparison with other diagnostic modalities in breast cancer including technical principles and clinical experiences. In comparison with 2D mammograms, DBT is better able to distinguish a lesion from overlapping breast tissue and to improve detection performance especially of masses and makes it possible to clarify the location or distribution of microcalcifications. In comparison with CE MRI, the diagnostic performance of DBT is comparable or better in some cases. CE MRI has an advantage in detecting cancerous lesions and extensive intraductal component or daughter lesions associated with the main cancerous lesions. However, by CE MRI, the differentiation between a small cancerous or benign lesion is sometimes difficult. On the other hand, the pixel size of DBT is much smaller than MRI and has an advantage regarding the morphological diagnostic approach. In comparison with US, the diagnostic performance of DBT is comparable in detecting mass lesions. In addition, DBT has advantages in detecting the lesions with microcalcifications, a large area, or multi-focal lesions in comparison with US.

Learning Objectives:

1. To understand image acquisition and image reconstruction principles of DBT

2. To understand the advantages and disadvantages of DBT compared to other diagnostic modalities

3. To understand the findings of DBT with reference to pathological diagnosis and 2D mammograms

Impact of imaging on staging, planning and treatment delivery in curative breast cancer radiotherapy

S. Bodis; Aarau/CH

High-precision radiotherapy $(\mathrm{RT})$ and vigorous $\mathrm{QA}$ are mandatory for curative breast cancer (BC) RT concepts. Modern imaging is mandatory both for timely staging, RT-planning and guidance of RT-delivery. Available three-dimensional diagnostic tools for local and loco-regional breast imaging are e.g. CT, MRI and PET. Fusion of all relevant diagnostic images with RT-planning images should be a routine process. There is room for improvements on all levels. In general, diagnostic and therapeutic radiation disciplines need to be better coordinated. Specifically, imaging processing for RT-planning and RT-delivery has to be more users friendly and robust. Streamlining und simplifying workflow is one important task for the future. Promising RT-planning tools from diagnostic 3D data sets (e.g. image guided breast biopsy, digital tomosynthesis, breast MRI and regional CT-PET) need to be tailored to the needs of radiation oncology planning (identical patient positioning for diagnosis and therapy, data processing like image fusion, data transfer and data storage). Radiation oncologists are lost in a swamp of diagnostic images which cannot be further processed, stand alone software and hardware from competing providers. Clinical radiation oncology study protocols and radiation oncology standards also need better description and definition of the most appropriate imaging methods for defined BC RT-concepts.

Learning Objectives:

1. Demonstrate and discuss workflow for RT-planning and RT-delivery with modern imaging

2. Future developments of imaging in curative breast cancer RT

3. Future opportunities for the link of diagnostic radiology and therapeutic radiotherapy

From control to prediction, moving forward in the field of neoadjuvant chemotherapy

R.M. Mann; Nijmegen/NL

While the use of neoadjuvant chemotherapy (NAC) historically was a last resort, it has now gained a place in the treatment of resectable and even relatively small breast cancers. Evaluation of response to NAC is a radiological challenge that is best tackled by MRI. Since NAC is by nature toxic, early proof of curative potentia is essential to prevent unnecessary harm to patients. Currently available tools however still focus on changes in size of the tumor and therapy is only altered in clear non-responders. Application of novel and improved MRI techniques such as farmacokinetic modeling, diffusion weighted imaging and MR spectroscopy, as well as PEM may allow earlier identification of responders and non-responders and even predict eventual response prior to the start of therapy. However, none of these modalities is by itself specific enough to alter current practices. It thus seems that we will need to rely on sophisticated computer algorithms for the combination of all available evidence in order to improve patient care.

Learning Objectives:

1. To define the current position of radiological imaging in the evaluation of patients treated with NAC. 
2. To understand the nature of new techniques for the evaluation of NAC.

3. To be able to give a global prediction of the probable response of a tumor to NAC.

Panel discussion

$14: 30-16: 00$

Studio 2012

jointly organised by Siemens Healthcare and Bayer Healthcare

\section{SY 4}

Programme not available by date of print

\section{Friday, March 2}

$12: 30-13: 30$

Room D1

organised by Bayer Healthcare

SY 5

Leading the way in contrast-enhanced MR imaging

Moderator:

F. Sardanelli; San Donato/IT

Choice of contrast in CNS MRI: what is the impact?

N. Anzalone; Milan/IT

More than 10 years of experience with Gadovist ${ }^{\circledR}$

F. Sardanelli; San Donato/IT

VALUE of Primovist ${ }^{\circledR}$ MRI vs CE-MRI and MDCT for staging of patients with liver metastases

C.J. Zech; Munich/DE

$12: 30-13: 30$

organised by GE Healthcare

SY 6

High-field MRI in a new light

Moderator:

A. van der Lugt; Rotterdam/NL

Humanising radiology

J. Coumans; Waukesha, WI/US

3T MRI: new trends in cerebral vascular imaging

J. $\underline{\text { Hodel; Paris/FR }}$

PET/MR vs PET/CT: the clinical reality check starts

G.K. von Schulthess; Zurich/CH

$12: 30-13: 30$

Room G/H

organised by Bracco

SY 7

Strategies for radiation dose reduction in contrastenhanced CT

Moderator:

C. Catalano; Rome/IT

How to marry scanner and contrast variables to maximise radiation

dose reduction

M. Prokop; Nijmegen/NL

Coronary CTA and beyond: new evidence and recommendations

H.-C. Becker; Munich/DE

Protocols for body CT: tips and tricks

A.J. Aschoff; Kempten/DE

Interactive discussion

$12: 30-13: 30$

Room I/K

organised by Philips Healthcare

SY 8

Programme not available by date of print 


\section{Saturday, March 3}

$12: 30-13: 30$

Room D1

jointly organised by Siemens Healthcare and

Bayer Healthcare

\section{SY 9}

\section{Synergies in CT - for better patient care}

Moderator:

J.E. Wildberger; Maastricht/NL

Programme not available by date of print

$12: 30-13: 30$

Room E1

\section{organised by Guerbet}

\section{SY 10}

\section{Vessel and muscle in coronary heart disease}

Moderator:

S. Plein; Leeds/UK

Introduction

S. Plein; Leeds/UK

Updated developments in coronary MSCT in patients with atrial fibrillation M. Dewey; Berlin/DE

In patients with atrial fibrillation coronary CT angiography is not reliably performed with high nondiagnostic rates in the per-patient analyses. Using 320-row volume CT one can scan the entire heart in a single rotation during just one heart beat (Dewey et al. Circulation 2009). This has great potential to enable reliable imaging also in patients with atrial fibrillation who are often sent for invasive angiography simply to rule out coronary artery disease. The hypothesis of the present trial will thus be that coronary CT angiography using 320 simultaneous detector rows using iobitridol 350 as the contrast agent results in high accuracy for detection of coronary stenoses in comparison to conventional coronary angiography in all patients (head-to-head comparison). Also interobserver variability, image quality, contrast agent amount, and radiation exposure required for volume $\mathrm{CT}$ and conventional coronary angiography will be intraindividually compared in all patients as the primary secondary objectives.

Detection of occult myocardial infarcts and coronary stenosis in asymptomatic diabetic patients

T.-H. Lim; Seoul/KR

Cardiac computed tomography angiography (CCTA) has emerged as a valid alternative imaging modality for the evaluation of patients with known or occult CAD. With the advent of MDCT technology, CCTA has the potential to provide comprehensive information regarding the location, severity, and characteristics of atherosclerotic plaque. Recently, CCTA has been suggested as a potential tool for providing a better insight about the occult CAD in asymptomatic individuals including asymptomatic patients with type 2 diabetes. Cardiac magnetic resonance (CMR) imaging can detect and characterize myocardial scar that is missed by ECG, conventional wall motion, or nuclear scintigraphic techniques but is associated with important cardiac events, including death and recurrent myocardial infarction. Furthermore, CMR imaging can characterize occult myocardial scar on delay-enhanced MR (DE-MRI) in diabetic patients without clinical evidence of myocardial infarction, and this occult myocardial scar showed strong association with major adverse cardiac events (MACE) and mortality hazards that is incremental to clinical, ECG, and left ventricular function combined. The ACCREDIT study (Assessment with Cardiac Computed Tomography Angiography and Magnetic Resonance Imaging in Patients with with Type 2 Diabetes for Detection of Unrecognized Myocardial Scar in Subclinical Coronary Atheroslerosis) were planned and performed for two purposes; 1 . to prospectively investigate the prevalence and predictors of occult myocardial scar on CMR imaging in subclinical atherosclerosis with type 2 Diabetes on CCTA or with traditional risk factors for CAD, and 2. to test the hypothesis whether subclinical coronary atherosclerosis on CCTA is associated with and myocardial scar on DE-MRI to provide strong prognostic value in asymptomatic patients with type 2 diabetes. The preliminary results of the ACCREDIT study will be provided in this lecture.
Why not having both with cardiac hybrid imaging?

O. Gämperli; Zurich/CH

Purpose: Computed tomography coronary angiography (CTCA) and myocardial perfusion imaging techniques (single photon emission computed tomography, SPECT, or positron emission tomography, PET) are established non-invasive modalities for the diagnosis of coronary artery disease (CAD). Material and Method: Cardiac hybrid imaging consists of the combination (or ,fusion") of both modalities and allows obtaining complementary morphological (coronary anatomy, stenoses) and functional (myocardial perfusion) information in a single setting. However, hybrid cardiac imaging has also generated controversy with regard to which patients should undergo such integrated examinations for clinical effectiveness and minimization of costs and radiation dose. The feasibility and clinical value of hybrid imaging has been documented in small cohort studies and selected series of patients. Results: Hybrid imaging appears to offer superior diagnostic and prognostic information compared with stand-alone or side-by-side interpretation of data sets. Particularly in patients with multivessel disease, the hybrid approach allows identification of flow-limiting coronary lesions and thereby provides useful information for the planning of revascularization procedures. Furthermore, integration of the detailed anatomical information from CTCA with the high molecular sensitivity of SPECT and PET may be useful to evaluate targeted molecular and cellular abnormalities in the future. Conclusion: While currently still restricted to specialized cardiac centres, the ongoing efforts to reduce radiation exposure and the increasing clinical interest will further pave the way for an increasing use of cardiac hybrid imaging in clinical practice.

Questions and answers

S. Plein; Leeds/UK

$12: 30-13: 30$ Room F1

\section{organised by Hitachi}

SY 11

Programme not available by date of print

$12: 30-13: 30$

Room F2

organised by Philips Healthcare

SY 12

Programme not available by date of print

$12: 30-13: 30$

Room I/K

organised by Bracco

SY 13

Programme not available by date of print

$12: 30-13: 30$

Room L/M

organised by Philips Healthcare

SY 14

\section{Advances in radiology ultrasound using the} iU22 xMatrix

Moderator:

E. Leen; London/UK

xMatrix, a new diagnostic tool for abdominal ultrasound

D.A. Clevert; Munich/DE

Advancements in musculoskeletal ultrasound

C. Martinoli; Genoa/IT

New advances in liver elastography

O. Kolokythas; Seattle, WA/US 


\section{organised by Philips Healthcare}

\section{SY 15}

\section{Shaping the future of breast cancer screening}

Moderators:

M. Danielsson; Solna/SE

H. Ringertz; Linköping/SE

Single-shot spectral mammography in clinical practice

M.G. Wallis; Cambridge/UK

A challenge within mammography is to improve cancer detection while at the same time reducing the rate of unnecessary recalls. In CT it is today common practice to use dual energy methods to discriminate between different tissues. Phantom work using a new photon counting spectral detector from Philips suggests it might also be possible to use similar methods in mammography. One example presented here is to assess the water content of circular lesions, thereby adding supportive information to radiologists when making a decision about recall or not. A study aiming at providing a firm scientific base to develop these ideas is now ongoing and the information obtained will be used to optimize the detector software and determine the spectral fingerprint of cysts.

Breast density measurement - risk assessment in screening S. Molloi; Irvine, CA/US

It is known that dense breasts imply a higher risk for developing breast cancer. Work is ongoing to find objective ways of measuring breast density, and in the future tie that to risk assessment. The vision is that all women shall have breast density measurements done at screening and based on that the radiologists can decide who is at risk and needs shorter screening intervals and also possible additional examinations with for example ultrasound. Dual energy mammography was used to measure breast density. In this study postmortem breasts were imaged and a comparison was made between dual energy mammography, MRI and CT, with chemical decomposition as gold standard. Philips' photon counting detector has the ability to distinguish high energy photons from photons with low energy and a study has been conducted looking at the potential for spectral mammography to accurately measure breast density without the additional radiation dose.

Benefits of dose reduction in mammography

E. Pisano; Charleston, SC/US

Breast cancer screening is a well-established method for reducing mortality in women, because cancers can be detected at an early stage with a far better prognosis. For a radiologist the image quality is the most important aspect and the determining factor for finding breast cancer at an early stage. However, breast tissue is sensitive to radiation and the risk of $x$-ray induced cancer is relative to lifetime exposure, therefore the ALARA principle is a regulatory requirement for all radiation safety programs. A non-inferiority study has been conducted comparing Philips MicroDose to a predicate device showing that dose reduction does not necessarily imply compromising image quality.

\section{organised by Siemens Healthcare}

SY 18

\section{Creating the future of MRI}

Moderator:

C.D. Claussen; Tübingen/DE

Welcome creating the future of MRI

C. Zindel; Erlangen/DE

3T MRI - between a luxury good and clinical necessity

A. McKenna-Küttner; Frankfurt/DE

How to address workflow challenges in a broad spectrum MRIservice: first experiences with Dot

C. Bremer; Münster/DE

Biograph mMR - clinical reality of simultaneous MR-PET and future realm of possibilities

C.D. Claussen; Tübingen/DE

$14: 00-15: 30$

Room E1

organised by Toshiba

SY 19

Programme not available by date of print

$14: 00-15: 30$

Room N/O

organised by GE Healthcare

SY 20

From in-vivo to in-vitro, enabling personalised cancer care

Moderator:

R.C. Sigal; Velizy/FR

Is there any advantage of volume navigation and needle tracking

techniques in the management of oncology patients?

T. Albrecht; Berlin/DE

MRgFUS: a non-invasive approach to thermoablation A. Beck; Berlin/DE

The clinical role of molecular breast imaging

E. Even-Sapir Weizer; Tel Aviv/IL

\section{organised by Bracco}

SY 16

Programme not available by date of print 


\section{Sunday, March 4}

\section{organised by GE Healthcare}

\section{SY 21}

\author{
New paradigm in CT imaging capabilities and \\ patient care \\ Moderator: \\ J. de Mey; Brussels/BE
}

Introduction

J. de Mey; Brussels/BE

Preserving diagnostic image quality with new $\mathrm{CT}$ reconstruction techniques and low concentration Iso-osmolar contrast media J.-L. Sablayrolles; St. Denis/FR

Purpose: The purpose of this study was to investigate and demonstrate that with image reconstruction techniques such as $A S i R \circledast$ and $V e o ß$, diagnostic image quality is maintained in patients scanned at lower $\mathrm{kVp}$ and injected with low concentration Visipaque ${ }^{\circledR}$ compared to lomeron ${ }^{\circledR} 400 \mathrm{mgl} / \mathrm{ml}$. Method and Materials: Visipaque ${ }^{\circledR} 270$ or $320 \mathrm{mgl} / \mathrm{ml}$ (GE Healthcare) was injected at $3.5 \mathrm{ml} / \mathrm{s}$ into a semianthropomorphic dynamic liver phantom (QRM, Germany) and CT scans were performed using a HD 750 CT scanner (GE Healthcare) at 80,100 or $120 \mathrm{kVp}$ at fixed $\mathrm{mA}(10-500 \mathrm{~mA}$ ) to obtain arterial (35s), arterial / portal (45s) and portal (70s) phase data. Similarly, abdominal CT was performed on patients after injection of Visipaque ${ }^{\circledR} 270$ or $320 \mathrm{mgl} / \mathrm{ml}$ or lomeron $\AA 400 \mathrm{mgl} / \mathrm{ml}(2 \mathrm{cc} / \mathrm{kg})$ at $3.5 \mathrm{ml} / \mathrm{s}$. CT scans were performed using an HD750 CT at 80, 100 and $120 \mathrm{kVp}$ to obtain arterial phase (25-35s) and portal phase (65-75s) data. Acquisition parameters were set according to patient's BMI. In both settings, projection data was reconstructed with FBP (standard) and two types of iterative reconstruction $A S I R \circledast-50 \%$ and $V e O \AA$. Results: In the dynamic phantom, marked stepwise reductions in noise were observed with both 120 and $100 \mathrm{kVp}$ scans after $A S I R \otimes-50 \%$ and Veo $\otimes$ reconstruction compared to FBP after injection of Visipaque at 270 or $320 \mathrm{mgl} / \mathrm{ml}$. By contrast, marked increases in "venous" enhancement was observed in $100 \mathrm{kVp}$ scans after injection of Visipaque ${ }^{\circledR} 270 \mathrm{mgl} / \mathrm{ml}$ compared to $120 \mathrm{kVp}$ scans after injection of Visipaque ${ }^{\circledR} 320 \mathrm{mgl} / \mathrm{ml}$. Clinical results matched the observations in the dynamic phantom. In patients, $100 \mathrm{kVp}$ combined with advanced reconstruction and Visipaque ${ }^{\circledR} 270 \mathrm{mgl} / \mathrm{ml}$ gave better contrast than $120 \mathrm{kVp}$ with $\mathrm{FBP}$ and Visipaque ${ }^{\circledR}$ $320 \mathrm{mgl} / \mathrm{ml}$. Importantly, contrast enhancement with lomeron ${ }^{\circledR} 400 \mathrm{mgl} / \mathrm{ml}$ was not superior to Visipaque ${ }^{\circledR} 320 \mathrm{mgl} / \mathrm{ml}$ when used in conjuction with ASIR. Indeed, ASiR and Veo reconstructions improved overall image quality of parenchyma, edges and interfaces and vascular structures after reduction in $\mathrm{kVp}$ and iodine concentration. Conclusion: These data demonstrate that advanced reconstruction with $A S I R B$ or Veo ${ }^{\circledR}$ in combination with low $\mathrm{kVp}$ (80 or 100) and low concentration Visipaque ${ }^{\circledR}$ (270 or $320 \mathrm{mgl} / \mathrm{ml}$ ) delivers equivalent or better contrast enhancement and improved diagnostic images compared to lomeron® $400 \mathrm{mgl} / \mathrm{ml}$ without impairing noise. Such improved contrast enhancement at low iodine concentrations and/or lower radiation dose represents a clear opportunity to improve patient care in CT.

Dose in paediatric imaging: how low can we go?

C. Ernst; Brussels/BE

Radiation dose is a major concern in computed tomography (CT) imaging, especially for sensitive organs or populations. A recent survey highlighted that only $43 \%$ of facilities adjusted CT techniques for children, whilst another survey found that $80 \%$ of CT studies in children were not managed by paediatric radiologists. In recent years Iterative reconstruction techniques have enabled a substantial reduction of radiation dose during CT scans. Several users reported a dose reduction that could reach $50 \%$, in some specific cases even $65 \%$, in pediatric imaging with ASiR iterative reconstruction, which models noise root cause in the reconstruction process. New techniques allow to model the image chain in the reconstruction process, leading to an increased spatial resolution at lower dose. GE Healthcare made recently this techniques available in clinical practice with the Veo product introduction. A series of pediatric patients presenting recurrent diseases (cystic fibrosis or lymphoma) were scanned and reconstructed with Veo. A dose reduction of $70 \%$ was noticed compared to FBP reconstruction and depending on the type of exam even a dose reduction of $85 \%$ was obtained without image quality loss. On some CT thorax examinations diagnostic quality images could be obtained with a dose of $0.05 \mathrm{mSv}$ wich, after further investigation, could provide new opportunities to replace conventional XRay exams by CT exams in certain indications. In summary, Veo enabled to reach dose reduction of $70-85 \%$ in paediatric examinations while maintaining and even improving image quality, depending on the type of exam. Dose reduction up to $90 \%$ and more was reached providing diagnostic quality images. This is of major importance in paediatric population which is known to be highly sensitive to radiation dose.

New advances in cardiovascular CT to lower dose and improve diagnosis G. Pontone; Milan/IT

The anatomical evaluation of the coronary arteries, the clinical indication for revascularization procedure and follow-up of patients has always been prerogative of invasive coronary angiography. However this technique has several disadvantages. First, it is invasive and associated with a not negligible incidence of adverse events. Second, it is expensive and last the information obtained via catheter-based coronary angiography pertains to the coronary arterial lumen alone. The introduction of multidetector computed tomography scanner associated with high-speed and ECG gated X-ray tube allowed an increase in spatial and temporal resolution such as to consider this technology a valuable alternative to the invasive coronary angiography. More recently, the use of low-dose technologies, such as SnapShot Pulse acquisition mode or ASiR (Adaptive Statistical Iterative Reconstruction) and High Definition scanner (Discovery CT750 HD, GE Healthcare) should allow to enlarge the indication of this test to middle age patients and to high risk patient. The aim of this lecture is to describe technical background, clinical applications of Cardiac CT and to present the new advances in Cardiovascular CT to lower dose and improve diagnosis.

Questions and answers

$12: 30-13: 30$

Room E1

organised by Toshiba

SY 22

Programme not available by date of print

$12: 30-13: 30$

Room G/H

organised by Philips Healthcare

SY 23

Programme not available by date of print

$12: 30-13: 30$

Room I/K

\section{organised by Bracco}

SY 24

Dynamic contrast enhanced ultrasound: an established radiation-free option for abdominal radiology

Moderator:

L. Solbiati; Busto Arsizio/IT

Introduction: 2011 and 2012, European and worldwide update of recommendations for CEUS

L. Solbiati; Busto Arsizio/IT

CEUS, a solution to reduce radiation exposure in abdominal imaging P.S. Sidhu; London/UK

Vuebox(TM) a new quantification software tool dedicated to CEUS F. Tranquart; Geneva/CH

Advances in interventional radiology with xMatrix CEUS and fusion imaging

E. Leen; London/UK 


\section{organised by Siemens Healthcare}

\section{SY 25}

Pioneering the future of ultrasound

Programme not available by date of print

$12: 30-13: 30$

Room N/O

organised by Philips Healthcare

SY 26

Programme not available by date of print

\section{Saturday, March 3}

10:00 - 11:00

Room 13

Mini Satellite Symposium organised by im3D

MSY 1

Enhancing diagnosis in CT-colonography: CAD in clinical practice, screening and training

Moderator:

A. Laghi; Latina/IT

CAD-radiologist interaction in clinical diagnosis: lessons from the CADIMPACT trial

D. Regge; Turin/IT

What we know about training radiologists: current status and future directions

P. Lefere; Roeselare/BE

Mass screening with CT-colonography: towards a patient friendly, computer-assisted examination

G. Iussich; Turin/IT

Final discussion 
